BMJ Open

Diabetes

Research

\& Care

\section{Pathobiology and Reversibility of Prediabetes in a Biracial Cohort (PROP-ABC) Study: design of lifestyle intervention}

Samuel Dagogo-Jack (D) , , ${ }^{1,2}$ Amy A Brewer, ${ }^{1}$ Ibiye Owei, ${ }^{1,2}$ Lindsey French, Nkiru Umekwe, ${ }^{2}$ Renate Rosenthal, ${ }^{3} \mathrm{Jim} \mathrm{Wan}{ }^{4}$
To cite: Dagogo-Jack S, Brewer AA, Owei I, et al. Pathobiology and Reversibility of Prediabetes in a Biracial Cohort (PROP-ABC) Study: design of lifestyle intervention. BMJ Open Diab Res Care 2020;8:e000899. doi:10.1136/ bmjdrc-2019-000899

Received 11 September 2019 Revised 23 April 2020 Accepted 18 May 2020

Check for updates

(c) Author(s) (or their employer(s)) 2020. Re-use permitted under CC BY-NC. No commercial re-use. See rights and permissions. Published by BMJ.

${ }^{1}$ General Clinical Research Center, The University of Tennessee Health Science Center, Memphis, Tennessee, USA

${ }^{2}$ Division of Endocrinology, Diabetes and Metabolism, The University of Tennessee Health Science Center, Memphis, Tennessee, USA

${ }^{3}$ Department of Psychiatry, The University of Tennessee Health Science Center, Memphis, Tennessee, USA

${ }^{4}$ Department of Preventive Medicine, The University of Tennessee Health Science Center, Memphis, Tennessee, USA

Correspondence to Dr Samuel Dagogo-Jack; sdj@uthsc.edu

\section{ABSTRACT}

Introduction Intensive lifestyle intervention (ILI) prevents progression from prediabetes to type 2 diabetes (T2D) but reversal of prediabetes is less well studied.

Research design and methods The overall objectives of the Pathobiology and Reversibility of Prediabetes in a Biracial Cohort (PROP-ABC) Study (ClinicalTrials. gov ID: NCT02027571) are to determine the natural history and reversibility of prediabetes. The study tests specific hypotheses on the patterns of progression to prediabetes among normoglycemic African-American (AA) and European-American (EA) offspring of parents with T2D; emergence of microvascular and macrovascular complications during transition from normal to impaired glucose regulation; significance of the 'metabolically healthy' obese phenotype; and effect of duration of the prediabetic state on its reversibility with lifestyle intervention. Participants who developed incident prediabetes were offered ILI and evaluated quarterly for 5 years. The primary outcome was restoration of normal glucose regulation (fasting plasma glucose $<100 \mathrm{mg} / \mathrm{dL}$ and two-hour plasma glucose $(2 \mathrm{hrPG})<140 \mathrm{mg} / \mathrm{dL})$. Results Of the 223 subjects enrolled in the PROP-ABC Study, 158 participants with incident prediabetes started ILI. The mean age was $53.3 \pm 9.28$ years; body mass index $30.6 \pm 6.70 \mathrm{~kg} / \mathrm{m}^{2} ; 70 \%$ were female, $52.4 \%$ AA and $47.6 \%$ EA. The ILI program used goal setting, weight-based calorie restriction, physical activity ( $180 \mathrm{~min} /$ week), selfmonitoring, and meal replacement. Monthly face-to-face (F2F) counseling sessions during the initial 6 months, and quarterly visits thereafter, were supplemented with electronic and postal contacts. Attendance at $\mathrm{F} 2 \mathrm{~F}$ sessions was highly correlated with weight loss $(r=0.98, p<0.0001)$. Meal replacement induced $\sim 5 \mathrm{~kg}$ weight loss within 3 months in participants with recrudescent weight pattern. Self-reported exercise minutes correlated with pedometer step counts $(r=0.47, p<0.0001)$.

Conclusion The PROP-ABC Study has demonstrated the feasibility of executing an ILI program designed to test reversibility of incident prediabetes in a biracial cohort.

\section{INTRODUCTION}

Estimates from the Centers for Disease Control indicate that $\sim 30$ million American adults have diabetes (predominantly type 2

\section{Significance of this study}

What is already known about this subject?

- Previous studies have shown that lifestyle intervention can prevent or delay type 2 diabetes in people with prediabetes.

- The primary outcome of the previous studies was prevention of progression from prediabetes to type 2 diabetes; enrollees were individuals with prevalent prediabetes who were identified during community screening efforts.

What are the new findings?

- The Pathobiology and Reversibility of Prediabetes in a Biracial Cohort Study applied intensive lifestyle intervention (ILI) in individuals with prospectively diagnosed incident prediabetes.

- Initially normoglycemic subjects were followed longitudinally for the occurrence of incident prediabetes and referred for ILI.

- The study targeted and enrolled high-risk subjects, namely African-Americans and European-Americans with parental history of type 2 diabetes.

- The ILI program used fewer clinic visits and a more intensive exercise target, compared with previous studies, along with a weight-based calorie restriction approach.

How might these results change the focus of research or clinical practice?

- Most people with prediabetes go undiagnosed and therefore, are not offered any lifestyle counseling.

- The present study documenting the successful execution of an ILI program in a diverse cohort of high-risk subjects with incident prediabetes should stimulate a more proactive screening, diagnostic, and lifestyle intervention behavior among clinicians.

diabetes, T2D), and $\sim 84$ million have prediabetes (defined as impaired glucose tolerance (IGT) or impaired fasting glucose (IFG)). ${ }^{1}$ The pathogenesis, mechanisms of the underlying defects, and complications of T2D have been well documented, but comparable data 
on the natural history of prediabetes are scant. ${ }^{23}$ Besides conferring increased risk for T2D, prediabetes is associated with vascular and neuropathic complications. ${ }^{4-6}$ Several randomized clinical trials have demonstrated the efficacy of lifestyle interventions in delaying the development of T2D in people with prediabetes. ${ }^{7-11}$ These landmark studies prespecified diabetes prevention, rather than reversal of prediabetes, as the primary outcome. ${ }^{7-10}$ Nonetheless, useful insight can be gleaned from the data on regression of prediabetes. In the Malmö study, $50 \%$ of the participants with initial IGT reverted to normal glucose tolerance with lifestyle modification during 6 years' follow-up. ${ }^{11}$ In the Diabetes Prevention Program (DPP), $\sim 40 \%$ of IGT subjects assigned to the lifestyle arm reverted to normal glucose regulation (NGR) during $\sim 3$ years' follow-up, compared with $\sim 20 \%$ in the placebo or metformin arms. ${ }^{712}$

As the prediabetic subjects in the Malmö, DPP and other studies were identified from screening operations, the chronicity of their prediabetes was unknown. ${ }^{7-11}$ In contrast, the Pathobiology of Prediabetes in a Biracial Cohort (POP-ABC) Study followed initially normoglycemic subjects quarterly for $\sim 5$ years, for the primary outcome of incident prediabetes (IFG or IGT). The design, baseline characteristics, and primary results of the POP-ABC Study have been published. ${ }^{13-15}$ Unlike the DPP and other landmark diabetes prevention studies, the POP-ABC Study was able to time the onset of prediabetes to within a 3-month window. Such near-precise ascertainment of the onset of prediabetes enables determination of the impact of duration of prediabetes on the efficacy of lifestyle interventions to induce reversion to NGR. The Pathobiology and Reversibility of Prediabetes in a Biracial Cohort (PROP-ABC) Study, a 5-year extension of the POP-ABC Study, was designed to test the time-dependent reversibility of prospectively diagnosed prediabetes, using intensive lifestyle intervention (ILI). Here, we present the design of the PROP-ABC Study and details of the ILI program.

\section{RESEARCH DESIGN AND METHODS \\ Design of the PROP-ABC Study}

Figure 1 summarizes the design of the PROP-ABC Study and its relationship to the precursor POP-ABC Study. The POP-ABC Study enrolled 376 initially normoglycemic (NGR) African-American (AA) and European-Americans (EA) offspring of parents with T2D and followed them quarterly for the occurrence of incident prediabetes over 5.5 years (September 2006 until March 2012).$^{13-15}$ Being a natural history study, participants did not receive any behavioral or pharmacological intervention. Reports from the POP-ABC Study have identified several predictors of progression from NGR to incident prediabetes in a diverse cohort. ${ }^{15-20}$

The 5-year extension study, PROP-ABC, was initiated in October 2013, and re-enrolled 223 of the original POP-ABC participants. The overall objectives of the PROP-ABC Study were to gain a fuller understanding of the natural history and predictors of early glucose abnormalities, determine the role of race/ethnicity, and to assess the reversibility of prediabetes. The study tests four specific hypotheses: (1) Among offspring of parents with T2D, early progression (within 5 years) from normal to impaired glucose regulation occurs in the highest-risk subjects independently of race, whereas late progression (5-10 years) displays racial disparities. (2) Microvascular complications, peripheral vascular disease, and endothelial dysfunction manifest during transition from normal to impaired glucose regulation. (3) The 'metabolically

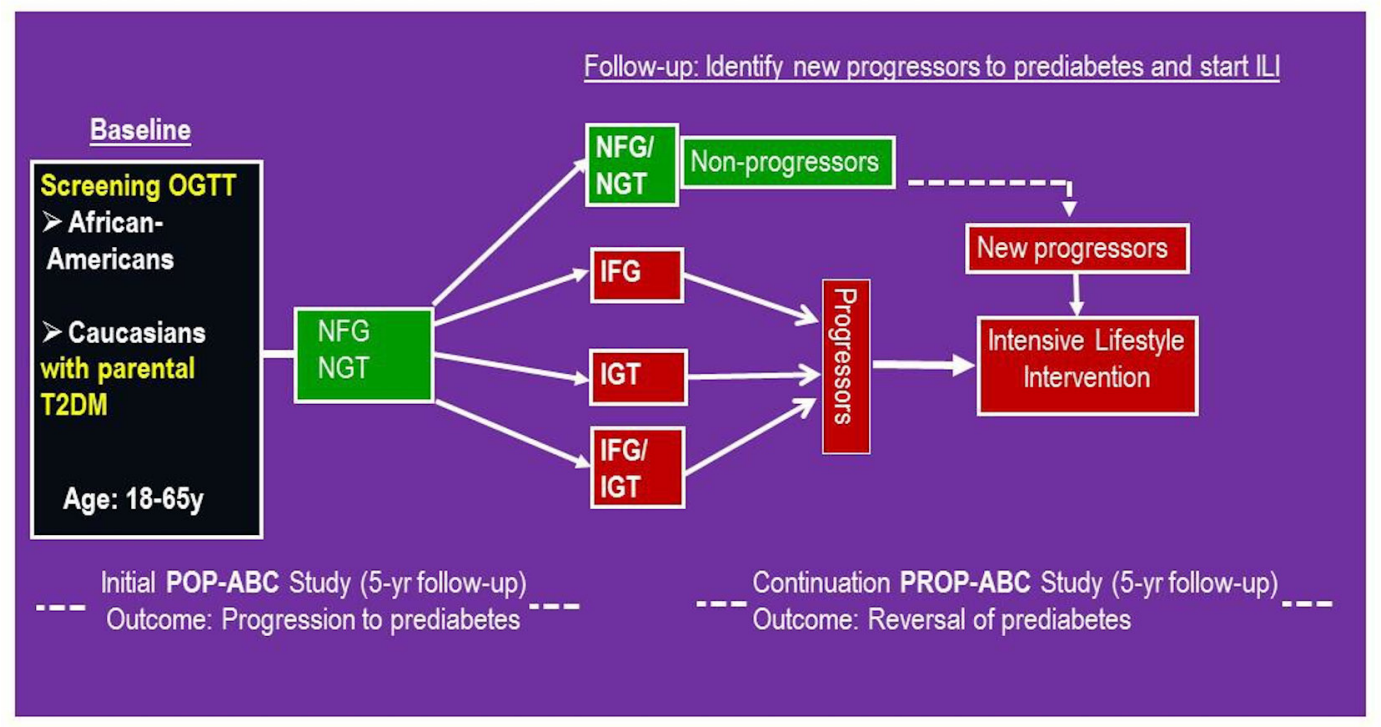

Figure 1 Schematic showing design of the Pathobiology of Prediabetes in a Biracial Cohort (POP-ABC) Study and the Pathobiology and Reversibility of Prediabetes in a Biracial Cohort (PROP-ABC) Study. IFG, impaired fasting glucose; IGT, impaired glucose tolerance; ILI, intensive lifestyle intervention; NFG, normal fasting glucose; NGT, normal glucose tolerance; OGTT, oral glucose tolerance test; T2DM, type 2 diabetes mellitus. 
healthy' insulin-sensitive obese phenotype displays racial disparities in its association with cardiometabolic risk factors and incident dysglycemia. (4) Duration of the prediabetic state determines the efficacy of ILI on the reversal of prediabetes and restoration of NGR.

We defined duration of prediabetes as the interval from date of confirmed prediabetes to the date of initiation of ILI. To assess the reversibility of prediabetes, participants who developed incident prediabetes during the POP-ABC phase, and those with newly occurring prediabetes during follow-up in the PROP-ABC phase, were offered ILI and evaluated quarterly for 5 years. The primary outcome was restoration of NGR (fasting plasma glucose $<100 \mathrm{mg}$ / $\mathrm{dL}$ and two-hour plasma glucose $(2 \mathrm{hrPG})<140 \mathrm{mg} / \mathrm{dL})$. Assessments included anthropometry, oral glucose tolerance test (OGTT), body composition, lipid profile, insulin sensitivity, insulin secretion, digital retinal photography, neuropathy testing, albumin excretion and endothelial function. All participants gave written informed consent prior to initiation of study, which was conducted at the General Clinical Research Center (GCRC) in accordance with the principles of the Helsinki Declaration.

\section{Overview of the lifestyle intervention}

The goal of the ILI program was to achieve a weight loss of $\geq 10 \%$ from baseline weight through calorie reduction and increased physical activity (180 minutes/week) among participants with incident prediabetes. The weight loss goal was higher than the 7\% target of the DPP but same as the goal in the Look AHEAD Study. ${ }^{7122}$ The physical activity target was higher than the 150 minutes/ week and 175 minutes/week targets of the DPP and Look AHEAD Studies, respectively. These higher weight loss and physical activity goals were selected to test their efficacy in reversing prediabetes, not merely prevention of progression to diabetes.

The ILI program was delivered by two registered dietitians with pertinent training and experience in lifestyle intervention in people with prediabetes and diabetes. Psychological support was provided by the study behaviorist (Dr. Rosenthal), a professor of clinical psychology, who had previously served in a similar role for the Look AHEAD Study. Counseling sessions with the study behaviorist covered such topics as role of life stressors in driving unhealthy habits (such as smoking, alcohol, drugs, overeating) that lay the groundwork for disease, and discussed strategies for managing stress, maintaining healthy habits, and optimizing self-awareness and selfcare. Routine sessions with the nutritionists also explored problem areas and provided emotional support.

\section{Clinic visits}

Our ILI program was delivered during monthly face-toface (F2F) counseling sessions for the initial 6 months (core sessions), followed by quarterly visits for the remainder of the study duration. The ILI combined group and individual counseling, and our on-site F2F contact frequency was less intensive than the DPP or
Table 1 Topics covered and incentives distributed during core sessions

\begin{tabular}{|c|c|}
\hline Main topics & Incentives \\
\hline $\begin{array}{l}\text { What is prediabetes? } \\
\text { Importance of lifestyle } \\
\text { management } \\
\text { Tracking calories for weight loss } \\
\text { Promoting exercise habits }\end{array}$ & $\begin{array}{l}\text { PROP-ABC logo } \\
\text { Tote bag } \\
\text { Notebook } \\
\text { Calorie King book } \\
\text { PROP logo pen }\end{array}$ \\
\hline $\begin{array}{l}\text { Emotional eating } \\
\text { How to eat fewer calories/ } \\
\text { volumetrics } \\
\text { Using your pedometer }\end{array}$ & Pedometer \\
\hline $\begin{array}{l}\text { What is BMI? } \\
\text { Healthy strategies in restaurants } \\
\text { Ask for what you need } \\
\text { Adding steps to your day }\end{array}$ & $\begin{array}{l}\text { PROP-ABC logo water } \\
\text { bottle }\end{array}$ \\
\hline $\begin{array}{l}\text { Handling the holidays, vacations, } \\
\text { special events } \\
\text { How are fats different? } \\
\text { Exercise safely in heat and cold }\end{array}$ & Hand weights \\
\hline $\begin{array}{l}\text { Savvy grocery store skills } \\
\text { Secrets to staying motivated } \\
\text { Making exercise enjoyable }\end{array}$ & $\begin{array}{l}\text { National Institutes of } \\
\text { Health }(\mathrm{NIH}) \text { exercise } \\
\text { handbook }\end{array}$ \\
\hline $\begin{array}{l}\text { Stress and your body } \\
\text { Eat easy: keeping it simple } \\
\text { Preventing relapse }\end{array}$ & PROP-ABC cookbook \\
\hline
\end{tabular}

BMI, body mass index; PROP-ABC, Pathobiology and Reversibility of Prediabetes in a Biracial Cohort.

the Look AHEAD Study (weekly sessions for 6 months, three sessions/month during months $7-12$, and monthly sessions in years 2-4). ${ }^{21}{ }^{22}$ Specifically, we omitted highfrequency weekly on-site visits during the initial 6 months, to improve community translation of our protocol.

\section{Core sessions}

The six core sessions were delivered to groups of 5-10 participants, using content adapted from the DPP and Look AHEAD Study lifestyle intervention strategies (table 1)..$^{21}$

Each monthly session lasted 2 hours with a $15 \mathrm{~min}$ break at the end of the first hour. All subjects were weighed privately before each session and computergenerated weight progress graphs were shared with each participant. Nutrition and exercise counseling sessions were presented by study dietitians and behavioral topics were presented by the study psychologist. The counseling strategies included educating participants on healthy eating patterns, calculation of total calories and fat grams consumed each day and discussing practical options for accruing the target exercise minutes. Participants were provided diaries for recording their daily calorie and fat intake and physical activity. Participants were encouraged to report progress with calorie restriction and exercise habits at each core session, and the peer group members and ILI staff facilitators provided constructive feedback as needed. 


\section{Quarterly (maintenance) sessions}

After completion of core sessions, participants were scheduled for ILI sessions every 3 months for the remainder of the PROP-ABC Study. Quarterly sessions lasted $90 \mathrm{~min}$, and were scheduled flexibly, allowing participants to select the most convenient meeting time and location. The quarterly sessions were delivered individually or in groups of 5-10 participants, at the convenience of participants. Educational topics were aimed at optimizing attainment of the weight and physical activity goals of the study. As was done during the core sessions, participants were weighed privately and given individual weight progress graphs. The records of food intake and exercise activities were collected at the end of each quarterly session.

Participants who missed a scheduled visit could reschedule to attend a make-up counseling session, which was delivered either individually or along with the next scheduled group, at the participant's convenience. Additionally, such participants were mailed handouts that summarized the counseling content of the missed visit.

\section{Additional modes of contact}

All ILI participants received a telephone call from study staff during the week following each core session, to provide support and to discuss privately any studyrelated questions. Telephone contact was also used to follow-up with participants who missed scheduled visits. Participants who disclosed during a study visit that they were experiencing life stresses received a follow-up telephone call from the study psychologist. During the first 6 months of ILI, participants were mailed two postcards each month. One postcard conveyed motivational and educational messages and the second was a reminder of the upcoming appointment date. During the maintenance period, participants received monthly postcards, conveying motivational and educational messages.

Other forms of communication included electronic mail and SMS text messaging. Email content included information on appointments and alerts about upcoming special campaigns. Participants who did not provide email addresses received the same information via regular mail. Text messaging was used less often, mostly to confirm appointments and request updates on participants. Regular newsletters mailed to participants gave highlights of the PROP-ABC Study.

\section{Detailed ILI modalities}

\section{Dietary intervention}

Participants received counseling on the benefits of healthy eating, regular meal times, and role of portion control in weight management. Emphasis was placed on reduction of fat intake and increased consumption of whole fruits, vegetables, lean meats, whole grains, dietary fiber and water. Participants were educated on the detriments of consuming high-calorie meals and sugar-flavored beverages. Like the Look AHEAD Study, we used a weight-based calculation to determine total daily calories and set fat intake target at $<30 \%$ of total calories. ${ }^{22}$ The calorie intake goals were $1200-1500 \mathrm{kcal} /$ day (40-50 gm fat) for persons with initial weight $<250 \mathrm{lbs}$ $(<113.4 \mathrm{~kg})$ and $1500-1800 \mathrm{kcal} /$ day $(50-60 \mathrm{gm}$ fat $)$ for those with initial weight $>250 \mathrm{lbs} .{ }^{22}$ The ILI dietary plan also recommended replacing saturated fat with healthier mono-unsaturated and poly-unsaturated fats. Participants received a nutrition reference book (Food Nutrition Facts and Free Calorie Counter, obtained from CalorieKing www. calorieking.com/us/en/) and were trained on using it to guide daily intake of calories and fat.

\section{Physical activity}

Our physical activity goal of $180 \mathrm{~min} /$ week is slightly higher than the Look AHEAD Study target of $175 \mathrm{~min} /$ week and is based on the finding that higher levels of physical activity improve weight loss maintenance. ${ }^{22} 23$ Participants were encouraged to distribute their activity over at least 3-6 days per week. Moderate-intensity physical activity was encouraged; brisk walking was the typical form of activity adopted by most participants. Participants were given a pedometer and encouraged to gradually increase their steps to 10000 or more daily. Some subjects preferred to use smartphone apps, instead of pedometer, for step counting.

\section{Self-monitoring}

Self-monitoring is an important adjunct to achieving and maintaining weight loss. ${ }^{721} 24$ All ILI participants recorded their daily dietary intake and exercise effort in diaries provided by the study. Participants recorded the types of leisure-time physical activity and number of minutes spent on each activity daily. Using the nutrition reference guide provided, participants also recorded their daily intake of calories and fat grams. The data on dietary intake and physical activity recorded in diaries along with records of steps obtained from pedometers and smartphone apps were reviewed by study staff during scheduled visits.

\section{Smartphone apps}

The use of smartphone apps to track dietary intake was encouraged. Participants were introduced to MyFitnessPal at the first core session and were provided support in setting up the application on their phone, if they desired. ${ }^{25}$ MyFitnessPal was selected because of its large searchable food database and free access. Smartphone apps allowed subjects to email or print out food intake summaries that could be reviewed with study dietitians during visits.

\section{Tool box}

Participants unable to meet weight goals were offered 'tool-box' approaches (focused on problem solving, motivational interviewing, behavioral contracts, provision of walking shoes, a cookbook, and meal replacements (MRs)). A behaviorist in the study team met individually with participants who were experiencing life stresses that could impact their commitment to the study. 
Miscellaneous interventions

Meal replacements

Use of MRs was introduced as a means of reversing weight regain or promoting weight loss in participants who had not met their weight goal after 6-12 months of ILI. Participants were selected for MR at the discretion of intervention staff, and no specific amount of weight regain was set as a trigger for initiation of MR. Our MR protocol was patterned after a previous study that demonstrated benefit to patients with prediabetes. ${ }^{26}$

The protocol involved replacing two meals with two shakes and two protein bars each day as part of a calorie-restricted meal plan (macronutrient distribution approximated $40 \%$ carbohydrate, $30 \%$ protein and $30 \%$ fat). The options for shakes were Glucerna Hunger Smart (15 gm protein), Atkins Shakes (15 gm protein), and Slim Fast Advanced Nutrition Shakes (20 gm protein). We selected Zone bars for MR due to their wide local availability and large variety of flavor options. To maximize adherence, participants underwent a blind taste test before selecting a preferred shake brand. All MR participants were encouraged to pursue the physical activity target of 180 min per week.

\section{Special campaigns}

The PROP-ABC Study held four special campaigns for the ILI participants.

\section{Holiday weight maintenance challenge}

During the winter holiday period (Thanksgiving through Christmas and New Year), participants were invited to join a voluntary 'weight challenge' contest aimed at maintaining pre-Thanksgiving weight throughout the winter holidays. Participants who joined the contest submitted to a pre-Thanksgiving 'weigh-in' and a post New Year's 'weigh-out' by study staff. Participants who completed the weigh-in and weigh-out procedures and maintained preThanksgiving weight (or lost weight) received an exercise shirt embossed with the PROP-ABC logo.

\section{PROPulsion campaign}

The 'PROPulsion' campaign was a 3-month optional initiative aimed at reinforcing healthy lifestyle habits. During the campaign, participants received weekly postcards displaying inspirational quotes from US astronauts. Between early June and late August 2017, participants who enrolled in PROPulsion received three individualized in-person counseling sessions (1 month apart), during which they were asked to select two behaviors they would like to improve during the campaign. Those needing more weight change were encouraged to select a behavior that would promote weight loss as one of their goals. Individual, 1 hour, appointments were scheduled flexibly with the study dietitians, during which participants received an updated weight progress graph. A 'PROPulsion' campaign T-shirt was distributed to participants during the first visit, a microwave-safe portion plate was given at the second contact, and small kitchen items to prime vegetable consumption (paring knife, vegetable spiralizer, and cutting mats) were provided at the third visit.

\section{0-day self-weighing challenge}

The 90-day self-weighing challenge was initiated to exploit the reported beneficial effect of frequent self-weighing on maintenance of weight loss. ${ }^{24}$ Participants, who opted in, agreed to record their daily weight for 90 consecutive days and have their waist circumference measured by study personnel at the beginning and end of the 90-day period. Digital scales were provided to those who did not have any at home. All subjects who completed the challenge received a selection of microwavable frozen vegetable products.

\section{Rocks and Rolls}

The 'Rocks and Rolls' initiative was a 3 month-long campaign designed to rekindle physical activity efforts. There were two activity groups within the campaign: Rockers and Rollers. The Rockers elected to increase aerobic activity by following an individual running and strength-training schedule, whereas the Rollers preferred low-impact strength-training exercises in a group setting. The Rocker track was patterned after the 'None to Run' plan developed by Mark Kennedy, which provided instructions on slow initiation and gradual escalation of running time and distance along with strength training. ${ }^{27}$ Participants in the Roller track met every other week for 12 weeks, for group sessions that focused on low-impact exercises for each major area of the body. The sessions, led by ILI staff, began with a brief anatomy of the muscle groups being exercised and a demonstration of proper posture for safe exercise, followed by group exercises. The study dietitians contacted participants in each track at 3,7 , and 12 weeks, to review exercise logs.

\section{Additional strategies}

\section{Visual feedback on weight loss progress}

During each visit, ILI participants were provided computer-generated graphs illustrating cumulative weight progress compared with their study weight goal. These cumulative weigh history graphs were used during counseling sessions. The visual feedback proved popular, at least among some participants, who reported proudly displayed their progress graphs at visible locations at work or at home. Some participants also reported sharing the weight progress data with their primary care physicians.

\section{Awards and recognition}

All PROP-ABC participants made scheduled visits to the GCRC for data collection; subjects who had progressed to prediabetes and were enrolled in ILI subjects made quarterly visits and non-progressors who maintained normoglycemia were seen every 6 months. During these visits, study dietitians implemented brief encounters with ILI participants and obtained information on specific exercise and dietary practices. After reviewing the food and exercise habits, study dietitians assigned points for 
each desirable habit. Participants with the highest score received a symbolic Gold Medal, and others received Silver or Bronze Medal, depending on total score.

Additionally, ILI participants who reached their weight loss goals were recognized annually as 'PROP Stars' in announcements mailed to all ILI participants.

\section{Statistical analysis}

Data are presented as means \pm SD unless SEM is specified. The association between weight change during MR and putative predictors (age, sex, ethnicity and duration of MR) was analyzed using multiple regression. The relationship between F2F ILI sessions and weight loss, and that between self-reported exercise minutes and pedometer step counts, was analyzed using linear regression models and Pearson correlation. All analyses were conducted using SAS (V.9.4, Cary, North Carolina, USA). A value of $\mathrm{p}<0.05$ was accepted as statistically significant.

\section{RESULTS}

\section{Cohort characteristics}

A total of 158 study participants who developed incident prediabetes were referred for ILI. Among these, 135 subjects $(85.4 \%)$ completed the study and had evaluable 5 -year follow-up data and 23 subjects $(14.6 \%)$ were lost to follow-up (12 dropped out, 8 could not be reached, 2 relocated and 1 died from causes unrelated to the study). The participants were predominantly women $(70 \%)$ but the ethnic ratio was more even $(52.4 \%$ AA, $47.6 \%$ EA). The mean age was $53.3+9.28$ years and the mean body mass index was $30.6+6.70 \mathrm{~kg} / \mathrm{m}^{2}$. At diagnosis of incident prediabetes, the mean fasting plasma glucose was $106+9.89 \mathrm{mg} / \mathrm{dL}$ and 2-hour plasma glucose during oral glucose tolerance test was $153+31.9 \mathrm{mg} / \mathrm{dL}$.

\section{F2F counseling sessions}

All F2F ILI sessions per protocol (20 for the entire study) were completed by $42.5 \%$ of subjects; $13.3 \%$ attended $75 \%, 14.8 \%$ attended $50 \%$ and $18.3 \%$ attended $\leq 25 \%$ of expected sessions. Table 2 shows the annual breakdown of F2F sessions completed by ILI participants during the PROP-ABC Study. Approximately $70 \%$ of participants attended at least $50 \%$ of all scheduled F2F ILI sessions and $56 \%$ of subjects attended at least $75 \%$ of sessions. The percentage of study subjects who attended fewer than $25 \%$ of scheduled F2F ILI sessions increased progressively from $<5 \%$ at inception to $30 \%$ by the end of the 5-year study period (table 2).

Although all subjects who missed an F2F session were mailed the educational content, we observed that physical attendance at lifestyle counseling sessions was highly correlated with mean weight loss during the study $(\mathrm{r}=0.98, \mathrm{p}<0.0001)$ (figure 2A).

\section{Self-reported physical activity}

Participants recorded variable levels of physical activity in their self-monitoring logs. The lowest activity $(\leq 50 \mathrm{~min} /$ week) was reported by $19.7 \%$ of subjects and the highest level ( $>200 \mathrm{~min} /$ week) was reported by $22.2 \%$ of subjects; $29.3 \%$ reported $51-100 \mathrm{~min} /$ week and $28.8 \%$ reported 101-200 min/week of physical activity. Data downloaded from pedometers showed that participants took $6564 \pm 3053$ steps daily (median 6172 , IQR 4300 steps). There was a significant correlation between daily steps and self-reported exercise minutes $(\mathrm{r}=0.47, \mathrm{p}<0.0001)$.

\section{Special campaigns}

Table 3 shows the data on voluntary participation in the special campaign events. Overall, participation in these optional offerings ranged from $30 \%$ to $60 \%$ of eligible study subjects and completion rates ranged from $60 \%$ to $90 \%$. Weight maintenance or modest weight loss was achieved by most participants of the special campaigns, except for the 90-day self-weighing challenge and the Rocks and Rolls campaign, both of which showed mixed results (table 3 ).

\section{Non-physical contacts}

A total of 5524 email messages were transmitted to ILI participants during the 5-year study. As 158 subjects with incident prediabetes were initially enrolled in ILI, the average email communication was $\sim 35$ messages per subject. We made 1404 telephone calls (averaging $~ 9$ calls/subjects) and sent 6769 postal mails (averaging 49/ subject) during the same period. SMS text messaging was the least used mode of remote communication; we sent only 139 text messages $(<1 /$ subject $)$ during the study.

Table 2 Numbers and percentages of participants completing scheduled face-to-face intensive lifestyle intervention sessions

\begin{tabular}{lcccc}
\hline $\begin{array}{l}\text { Percentage of face-to-face } \\
\text { sessions attended }\end{array}$ & $\begin{array}{l}\text { Year 1-2 number } \\
\text { (\%) of participants }\end{array}$ & $\begin{array}{l}\text { Year 2-3 number } \\
\text { (\%) of participants }\end{array}$ & $\begin{array}{l}\text { Year 3-4 number } \\
\text { (\%) of participants }\end{array}$ & $\begin{array}{l}\text { Year 4-5 number } \\
\text { (\%) of participants }\end{array}$ \\
\hline $100 \%$ & $51(32.3 \%)$ & $68(44.7 \%)$ & $70(47.6 \%)$ & $61(45.2 \%)$ \\
\hline $75 \%$ & $28(17.7 \%)$ & $23(15.1 \%)$ & $18(12.2 \%)$ & $11(8.15 \%)$ \\
\hline $50 \%$ & $37(23.4 \%)$ & $25(16.5 \%)$ & $15(10.2 \%)$ & $12(8.89 \%)$ \\
$25 \%$ & $35(22.2 \%)$ & $11(7.24 \%)$ & $12(8.16 \%)$ & $10(7.41 \%)$ \\
$<25 \%$ & $7(4.43 \%)$ & $25(16.5 \%)$ & $32(21.8 \%)$ & $41(30.4 \%)$ \\
\hline Total participants & $158(100 \%)$ & $152(96.2 \%)$ & $147(93.0 \%)$ & $135(85.4 \%)$ \\
Dropout/inactive & 0 & $6(3.80 \%)$ & $11(7.00 \%)$ & $23(14.6 \%)$ \\
\hline
\end{tabular}


A

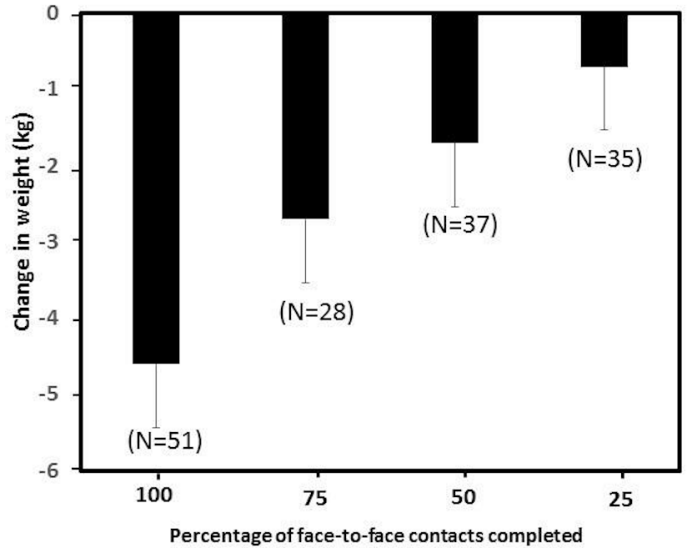

B

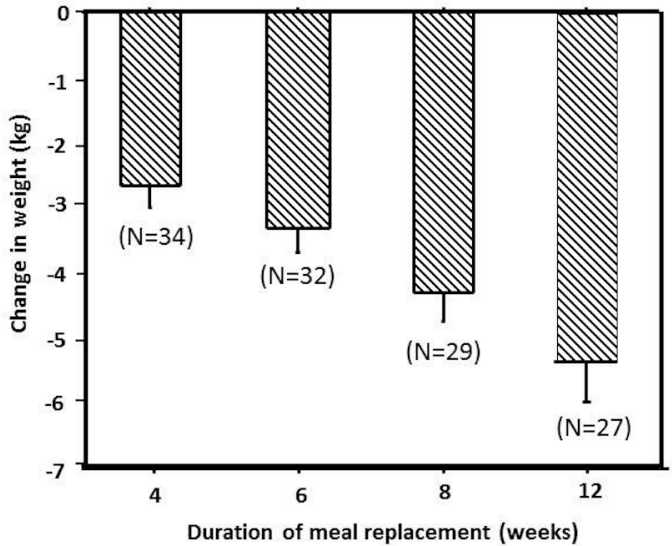

Figure 2 (A) Mean change in weight in relation to year 1 face-to-face contact frequency among 158 intensive lifestyle intervention participants. (B) Relationship between duration of meal replacement and 4-month change in body weight among participants who took meal replacement for at least 16 weeks. Change in weight was significantly correlated with face-to-face contact frequency $(r=0.98, p<0.0001)$ and duration of meal replacement $(r=0.77, p=0.001)$.

\section{Impact of MR}

We offered MR to selected participants who either were unable to meet weight goal after 6-12 months on standard ILI or regained weight after initial weight loss. The participants met weekly with research staff to collect replacement meals and undergo assessment. During the study, a total of 51 participants were offered $M R$, with variable acceptance and duration. The mean $( \pm \mathrm{SD})$ duration on MR was $21.0 \pm 18.4$ weeks (median 14 weeks, range 1-70 weeks). Figure $2 \mathrm{~B}$ shows the relationship between duration of MR and 4-month change in body weight among participants who took MR for at least 16 weeks. Progressive weight loss was evident within 4 weeks of initiation and was sustained throughout the period of MR. The mean weight loss was $2.68 \pm 1.51 \mathrm{~kg}$ at 4 weeks, $4.32 \pm 1.75 \mathrm{~kg}$ at 8 weeks and $5.34 \pm 2.43 \mathrm{~kg}$ at 12 weeks during MR (figure 2B). The duration of MR was significantly correlated with change in weight $(\mathrm{r}=0.77, \mathrm{p}=0.001)$ and waist circumference $(\mathrm{r}=0.55$. $\mathrm{p}=0.005)$. In a multiple regression model with terms for duration of MR, age, sex, and race, the duration of MR $(p=0.0014)$ was the only significant predictor of weight loss following MR.

\section{DISCUSSION}

As few studies have prespecified reversal of prediabetes as a primary end point, the goal of the PROP-ABC Study is to determine the effect of lifestyle intervention in reversing the prediabetes phenotype and restoring NGR. Emerging data indicate that people with prediabetes who revert to NGR, even if transiently, enjoy long-term protection from incident T2D and microvascular complications. ${ }^{28} 29$ A recent report from the Da Qing Study showed that prevention or delay of T2D among prediabetic subjects is associated with decreased mortality and vascular complications. ${ }^{30}$ These findings argue strongly in favor of restoration of NGR as a primary goal in people with prediabetes.

The PROP-ABC Study's ILI program was built on the well-known benefits of optimized nutrition, exercise, self-monitoring, and behavioral support on weight and glycemic outcomes in multiethnic populations. ${ }^{7} 212231-34$ The ILI program used individual and group counseling sessions, frequent physical and remote contacts, a structured initial core curriculum delivered monthly during the initial 6 months, and quarterly maintenance sessions. We also employed a 'toolbox' of strategies, including

Table 3 Proportion of 135 eligible intensive lifestyle intervention participants who joined optional special campaigns

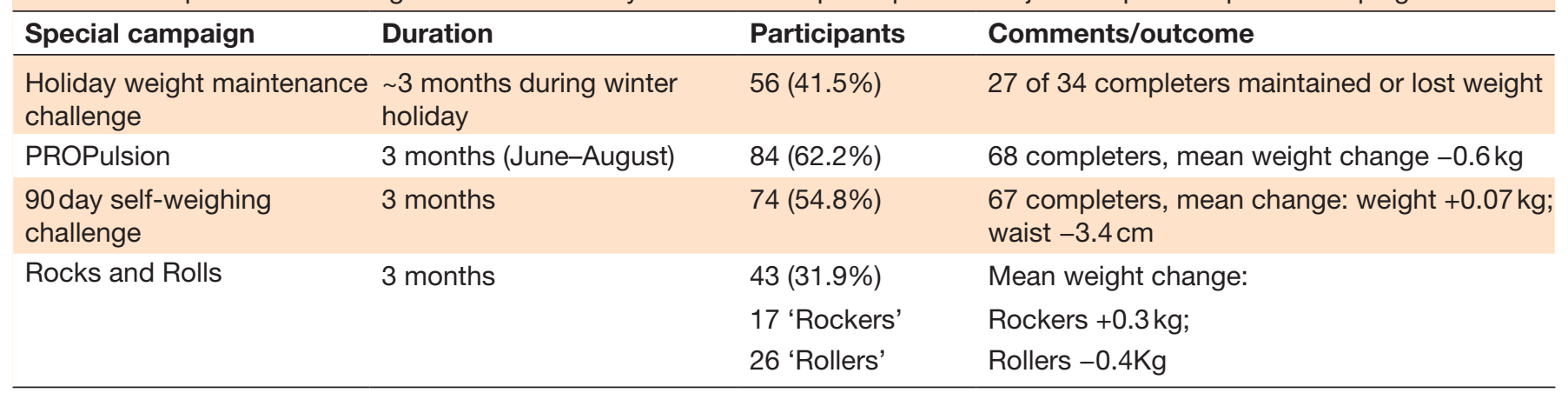


individual sessions with a behaviorist, during which adherence challenges and suboptimal or rebounding weight patterns were addressed. Additionally, we launched a variety of special campaigns throughout the study period, aimed at optimizing healthy behaviors.

The effects of these strategies on the primary outcome of the PROP-ABC Study (ie, reversal of prediabetes status) are being analyzed for a separate report. However, our experience in executing the ILI program allows us to make pertinent observations. Our PROP-ABC Study protocol had set a target of $20 \mathrm{~F} 2 \mathrm{~F}$ standard counseling sessions over 5 years for participants. Despite flexible scheduling, we observed that $70.6 \%$ of enrollees attended $50 \%$ or more of the sessions, $55.8 \%$ attended $75 \%$ and only $42.5 \%$ attended $100 \%$ of scheduled sessions. These findings reflect the real-world challenges of balancing work and life demands with active participation in clinical trials. Although all syllabus materials were mailed to participants who missed study visits, it is unclear whether the impact of mailed materials would equate to that of physical attendance. In fact, our analysis indicated a significant correlation between the percentage of F2F sessions attended and the magnitude of weight loss, consistent with previous reports from the Look AHEAD study. ${ }^{35} 36$

Clearly, a requirement of physical attendance at frequent F2F counseling would hamper large-scale translation of diabetes prevention practices for the more than 84 million US adults with prediabetes. Alternative lifestyle intervention delivery methods via the internet have been proposed. ${ }^{37}$ Comparing an internet-based intervention to in-person counseling sessions, some studies have observed similar efficacy in weight-loss maintenance, whereas others have found less efficacy on weight control with internet intervention compared with in-person counseling. ${ }^{38-41}$ The reports indicating variable efficacy of internet delivery of lifestyle interventions underscore the need for additional data that would enable prediction of responders to internet intervention. Pending the availability of such data, a mixed model of in-person counseling sessions, supplemented with remote delivery methods, is an appealing practical approach to lifestyle intervention for weight control and health promotion.

Based on self-report, nearly a quarter of participants exceeded the physical activity target of $180 \mathrm{~min} /$ week and most achieved a level of 51-200 min/week. Objective data from pedometers showed an average upright activity of 6500 steps/day, which correlated with self-reported exercise minutes. We observed that participants unable to reach or maintain weight goal responded to MR, consistent with published reports. ${ }^{22}{ }^{26}$ Our experience with the special campaigns showed that optional programs with specific themes could be attractive to some participants. The four different campaigns attracted some $30 \%-60 \%$ of participants. Notably, these campaigns all emphasized goalsetting, self-control and self-monitoring behavior, and were home based (except for the Roller track of Rocks and Rolls that involved low-impact group exercises).
The strengths of the PROP-ABC Study include the longitudinal design, rigorous ascertainment and timing of the occurrence of incident prediabetes end points, prespecification of reversal of prediabetes as the primary outcome, and enrollment of a biracial cohort of highrisk subjects. The prospective study design along with the ascertainment of incident prediabetes to a narrow of $\sim 3$ months enables testing of our hypothesis on the time-dependent reversibility of prediabetes. Despite these strengths, the PROP-ABC Study does have some limitations, including the relatively small sample size and restriction of entry to offspring of T2D parents. The latter does limit the generalizability of findings from the study. Also, for ethical reasons, we did not include an untreated group of individuals with prediabetes to serve as control. Furthermore, participants received several, although small, incentive items throughout the study. The design of our study does not permit determination of the potential impact of these incentive items on motivated behaviors, such as clinic attendance or weight loss. Arguably, if participants were significantly motivated by the incentives, that would limit large-scale translation of the study to the community, where resources for providing incentives may be limited.

In summary, the PROP-ABC Study has executed a 5-year ILI program in subjects at risk for T2D. Here, we have described elements of the lifestyle intervention program, including participation rates and weight response patterns. Ongoing analysis of the clinical and biochemical data generated by the study would provide information on the impact of our ILI program on the reversibility of prediabetes and glycemic classification after 5 years of intervention.

Contributors All authors materially participated in the research and article preparation and gave final approval for the version submitted. SD-J conceived of and designed the study, analyzed data, drafted manuscript; AAB, IO, LF, NU, RR collected data, reviewed and revised manuscript; JW performed statistical analysis, and reviewed and revised the manuscript.

Funding This work was supported by the National Institutes of Health grant number R01 DK067269.

Competing interests None declared.

Patient consent for publication Not required.

Ethics approval The PROP-ABC Study protocol (ClinicalTrials.gov ID: NCT02027571) was approved by the University of Tennessee Health Science Center Institutional Review Board (Approval number 12-01970-FB).

Provenance and peer review Not commissioned; externally peer reviewed.

Data availability statement All data relevant to the study are included in the article. Deidentified data are available for sharing upon reasonable request.

Open access This is an open access article distributed in accordance with the Creative Commons Attribution Non Commercial (CC BY-NC 4.0) license, which permits others to distribute, remix, adapt, build upon this work noncommercially, and license their derivative works on different terms, provided the original work is properly cited, appropriate credit is given, any changes made indicated, and the use is non-commercial. See: http://creativecommons.org/ licenses/by-nc/4.0/.

ORCID iD

Samuel Dagogo-Jack http://orcid.org/0000-0001-5318-9677 


\section{REFERENCES}

1 Centers for Disease Control and Prevention. New CDC report: more than 100 million Americans have diabetes or prediabetes. Available: https://www.cdc.gov/media/releases/2017/p0718-diabetes-report. html [Accessed 5 Sep 2019].

2 Defronzo RA. Banting lecture. From the triumvirate to the ominous octet: a new paradigm for the treatment of type 2 diabetes mellitus. Diabetes 2009;58:773-95.

3 Ebenibo S, Edeoga C, Wan J, et al. Glucoregulatory function among African Americans and European Americans with normal or prediabetic hemoglobin A1c levels. Metabolism 2014;63:767-72.

4 Brannick B, Wynn A, Dagogo-Jack S. Prediabetes as a toxic environment for the initiation of microvascular and macrovascular complications. Exp Biol Med 2016;241:1323-31.

5 Abdul-Ghani M, DeFronzo RA, Jayyousi A. Prediabetes and risk of diabetes and associated complications: impaired fasting glucose versus impaired glucose tolerance: does it matter? Curr Opin Clin Nutr Metab Care 2016;19:394-9.

6 Brannick B, Dagogo-Jack S. Prediabetes and cardiovascular disease: pathophysiology and interventions for prevention and risk reduction. Endocrinol Metab Clin North Am 2018;47:33-50.

7 Knowler WC, Barrett-Connor E, Fowler SE, et al. Reduction in the incidence of type 2 diabetes with lifestyle intervention or metformin. N Engl J Med 2002;346:393-403.

8 Pan XR, Li GW, Hu YH, et al. Effects of diet and exercise in preventing NIDDM in people with impaired glucose tolerance. The dA Qing IGT and diabetes study. Diabetes Care 1997;20:537-44.

9 Tuomilehto J, Lindström J, Eriksson JG, Valle TT, et al. Prevention of type 2 diabetes mellitus by changes in lifestyle among subjects with impaired glucose tolerance. N Engl J Med 2001;344:1343-50.

10 Ramachandran A, Snehalatha C, Mary S, et al. The Indian diabetes prevention programme shows that lifestyle modification and metformin prevent type 2 diabetes in Asian Indian subjects with impaired glucose tolerance (IDPP-1). Diabetologia 2006;49:289-97.

11 Eriksson KF, Lindgärde F. Prevention of type 2 (non-insulindependent) diabetes mellitus by diet and physical exercise. The 6-year Malmö feasibility study. Diabetologia 1991;34:891-8.

12 Perreault L, Kahn SE, Christophi CA, et al. Regression from prediabetes to normal glucose regulation in the diabetes prevention program. Diabetes Care 2009;32:1583-8.

13 Dagogo-Jack S, Edeoga C, Nyenwe E, et al. Pathobiology of prediabetes in a biracial cohort (POP-ABC): design and methods. Ethn Dis 2011;21:33-9.

14 Dagogo-Jack S, Edeoga C, Ebenibo S, et al. Pathobiology of prediabetes in a biracial cohort (POP-ABC) study: baseline characteristics of enrolled subjects. J Clin Endocrinol Metab 2013;98:120-8.

15 Dagogo-Jack S, Edeoga C, Ebenibo S, et al. Lack of racial disparity in incident prediabetes and glycemic progression among black and white offspring of parents with type 2 diabetes: the pathobiology of prediabetes in a biracial cohort (POP-ABC) study. J Clin Endocrinol Metab 2014:99:E1078-87.

16 Boucher AB, Adesanya EAO, Owei I, et al. Dietary habits and leisuretime physical activity in relation to adiposity, dyslipidemia, and incident dysglycemia in the pathobiology of prediabetes in a biracial cohort study. Metabolism 2015;64:1060-7.

17 Edeoga C, Owei I, Siwakoti K, et al. Relationships between blood pressure and blood glucose among offspring of parents with type 2 diabetes: prediction of incident dysglycemia in a biracial cohort. $J$ Diabetes Complications 2017;31:1580-6.

18 Jiang Y, Owei I, Wan J, et al. Adiponectin levels predict prediabetes risk: the pathobiology of prediabetes in a biracial cohort (POP-ABC) study. BMJ Open Diabetes Res Care 2016;4:e000194.

19 Owei I, Umekwe N, Wan J, et al. Plasma lipid levels predict dysglycemia in a biracial cohort of nondiabetic subjects: potential mechanisms. Exp Biol Med 2016;241:1961-7.
20 Owei I, Umekwe N, Stentz F, et al. Amino acid signature predictive of incident prediabetes: a case-control study nested within the longitudinal pathobiology of prediabetes in a biracial cohort. Metabolism 2019;98:76-83.

21 The Diabetes Prevention Program Research Group. The diabetes prevention program (DPP): description of lifestyle intervention. Diabetes Care 2002;25:2165-71.

22 Look AHEAD Research Group, Wadden TA, West DS, et al. The look ahead study: a description of the lifestyle intervention and the evidence supporting it. Obesity 2006;14:737-52.

23 Jeffery RW, Wing RR, Sherwood NE, et al. Physical activity and weight loss: does prescribing higher physical activity goals improve outcome? Am J Clin Nutr 2003;78:684-9.

24 Wing RR, Phelan S. Long-term weight loss maintenance. Am J Clin Nutr 2005;82:222S-5.

25 myfitnesspal. Fitness starts with what you eat. Available: https:// www.myfitnesspal.com/

26 König D, Kookhan S, Schaffner D, et al. A meal replacement regimen improves blood glucose levels in prediabetic healthy individuals with impaired fasting glucose. Nutrition 2014;30:1306-9.

27 Kennedy M. None to Run-Week 1 Workout 1. Available: https:// soundcloud.com/mark-kennedy-64/workout01

28 Perreault L, Pan Q, Mather KJ, et al. Effect of regression from prediabetes to normal glucose regulation on long-term reduction in diabetes risk: results from the diabetes prevention program outcomes study. The Lancet 2012;379:2243-51.

29 Perreault L, Pan Q, Schroeder EB, et al. Regression from prediabetes to normal glucose regulation and prevalence of microvascular disease in the diabetes prevention program outcomes study (DPPOS). Diabetes Care 2019;42:1809-15.

30 Gong Q, Zhang P, Wang J, et al. Morbidity and mortality after lifestyle intervention for people with impaired glucose tolerance: 30-year results of the dA Qing diabetes prevention outcome study. Lancet Diabetes Endocrinol 2019;7:452-61.

31 Pereira MA, Kriska AM, Day RD, et al. A randomized walking trial in postmenopausal women: effects on physical activity and health 10 years later. Arch Intern Med 1998;158:1695-701.

32 Perri MG, McAllister DA, Gange JJ, et al. Effects of four maintenance programs on the long-term management of obesity. J Consult Clin Psychol 1988;56:529-34.

33 McAuley PA, Artero EG, Sui X, et al. Fitness, fatness, and survival in adults with prediabetes. Diabetes Care 2014;37:529-36.

34 Johnson JL, Slentz CA, Ross LM, et al. Ten-year legacy effects of three eight-month exercise training programs on cardiometabolic health parameters. Front Physiol 2019;10:452.

35 Wadden TA, West DS, Neiberg $\mathrm{RH}$, et al. One-year weight losses in the Look AHEAD study: factors associated with success. Obesity 2009;17:713-22.

36 Wadden TA, Neiberg RH, Wing RR, et al. Four-year weight losses in the Look AHEAD study: factors associated with long-term success. Obesity 2011;19:1987-98.

37 Tate DF, Jackvony EH, Wing RR. Effects of Internet behavioral counseling on weight loss in adults at risk for type 2 diabetes: a randomized trial. JAMA 2003;289:1833-6.

38 Turk MW, Yang K, Hravnak M, et al. Randomized clinical trials of weight loss maintenance: a review. J Cardiovasc Nurs 2009;24:58-80.

39 Harvey-Berino J, Pintauro S, Buzzell P, et al. Effect of internet support on the long-term maintenance of weight loss. Obes Res 2004;12:320-9.

40 Harvey-Berino J, Pintauro S, Buzzell P, et al. Does using the Internet facilitate the maintenance of weight loss? Int J Obes 2002;26:1254-60.

41 Wing RR, Tate DF, Gorin AA, et al. A self-regulation program for maintenance of weight loss. N Engl J Med 2006;355:1563-71. 